\title{
On the axioms of the forces in the mechanics of rigid bodies
}

\author{
Géza Lámer $^{1 *}$ \\ ${ }^{1}$ University of Debrecen, glamer@eng.unideb.hu, Hungary
}

\begin{abstract}
Newton summarised knowledge related to forces in three axioms. The first and second ones define the mechanical state and motion of the examined body when there is no force or when force is exerted on the body. The third defines the law of action and reaction. Newton did not define it as separate axiom but assumed that forces are completely independent from each other. The statics applies four axioms. The first applies to the balance of two forces while the second one applies of three forces. The third axiom defines the relationships inside an equilibrium force system. The fourth one is the axiom of action and reaction. The two axiom systems are independent from each other. Further the independent axioms are applied in case of constraint forces: frictionless reaction force orthogonal on the forced surface, friction force acts in the direction of the motion, the deformation can be elastic, plastic and viscous.
\end{abstract}

\section{Introduction}

Newton defined his system of axioms in „Principia” to describe the motion of objects, I. Newton [1]. Newton describes the mechanical state of the body in the axiom system if no forces are acting upon it, and also defines how the magnitude and direction of force related with quantitative measures typical of the motion of the body when forces are acting upon it, and finally summarises how the magnitude and direction of forces relate when one body acts on a second body. Newton did not refer to the relation of two or more simultaneously occurring forces and how the joint effects of these could be determined. At the same time he provided clear instructions regarding these two questions: forces are independent from each other when they occur and their effects add up according to the parallelogram rule. Newton, as his work was concentrated on the motion of (point like) bodies, did not deal with the issue of balance.

Newton's axioms did not explicitly define that forces are vector quantities; this statement is discussed in Corollary 1. and 2.

The axioms of statics are commonly defined for the equilibrium state of rigid bodies. These axioms define the conditions of equilibrium for two or three forces, as well as the method of conversion of one equilibrium force system into another equilibrium force system. This latter also applies to equilibrium force systems acting upon bodies in non-equilibrium states. Finally, the (Newtonian) axiom of action-reaction is also classified as an axiom of statics, although the axiom system of statics does not deal with the occurrence of forces.

The axioms of statics do not explicitly define forces as vector quantities; this statement ensues from the axiom regarding the equilibrium of three forces.

Generally, neither of the axiom systems deals with the occurrence of forces, although both include the axiom, which clearly defines the number of forces and their relationship to each other which can be interpreted as the model of effects arising between the two bodies. The two axiom systems apply to two different mechanical systems: the first one is intended to describe the motion of (point like) bodies while the second one is intended to describe the equilibrium of rigid bodies.

The fact that any triplet forms a vector is not unambiguous. There is reference that the axioms records the attributes (commutativity, associativity) of vector addition when interpreting the vector spaces and attributes of simultaneous operations with vectors and real numbers, P.R: Halmos [2]. The fact that force is a vector quantity and the joint effect of more forces is equivalent to the effect of the vectorial sum of more forces does not ensue from the fact that force is used to describe the interaction of two bodies. The study examines the two axiom systems, then an axiom system is compiled which includes the attributes of forces arising in mechanics, the operations regarding the mechanical state of bodies, i.e. the „method of use" of force.

\section{The Newtonian axioms}

Newton's axioms apply to motion of bodies, more

\footnotetext{
Corresponding author: glamer@eng.unideb.hu
} 
specifically to the motion changes of bodies, I. Newton [1].

Before presenting the axioms, a definition of force, of body, and of mechanical state is required for which the axioms apply to.

Newton defines force as the effect acting upon the body. "Definition 4. Impressed force is the action exerted on a body to change its state either of resting or of moving uniformly straight forward.", I. Newton [1], p. 405.

Newton uses the term matter instead of body. "Definition 1.: Quantity of matter is a measure of matter that arises from its density and volume." (Presently referred to as the product, I. Newton [1], p. 403.

Newton defines the ,initial" mechanical state as rest or uniform motion. See i.e. Definition 4.

The Newtonian axioms are the following.

THE FIRST NEWTONIAN AXIOM (Law 1.): "Every body perseveres in its state of being at rest or of moving uniformly straight forward, except insofar as it is compelled to change its state by forces impressed", I. Newton [1], p. 416.

This axiom applies to the state when no forces are acting upon the body; in other words no other body is acting upon it.

THE SECOND NEWTONIAN AXIOM (Law 2.): "A changes in motion is proportional to the motive force impressed and takes place along the straight line in which that force impressed.", I. Newton [1], p. 416.

This axiom, for a force impressed on a body, is currently defined as follows: the acceleration (a) of an object arises in the same direction as the force $(\mathbf{F})$ causing it while its magnitude is proportional to the magnitude of the force and inversely proportional to the mass $(m)$ of that object.

This axiom describes the relationship between the data determining force (magnitude, direction and sign) and data describing the motion change of the body (magnitude, direction and sign). This also means that this axiom defines the data describing the force at the same time: magnitude, direction and sign. However, this axiom does not describe how force arises. This is because gravitational forces act upon objects in a Newtonian mechanical system and the value and direction of these forces is determined by the relative position, mass and universal laws of gravity. On the one hand, the second axiom describes that if force acts upon an object then it changes the motion of the object, on the other hand it also describes how the change of the motion can be calculated. The second axiom connects only the force and its consequence and it does not deal with the simultaneous effect of more forces. This has to be examined separately.

THE THIRD NEWTONIAN AXIOM (Low 3.): "To any action there is always an opposite and equal reactions; in other words, the actions of two bodies upon each other are always equal and opposite in direction.", I. Newton [1], p. 417.

This axiom defines that if an effect arises while two bodies act upon each other, then these are essentially two „effects”, or two forces. At the same time it also defines how the data describing two forces - magnitude, direction and sign - relate to each other. It does not provide explicit information of the line of action, but it covertly assumes identical line of action in the subsequent explanation.

This axiom characterises how the force arises. Implicitly, it „follows" the first axiom. The second axiom characterises the numeric value of force and its effect, i.e. effect of one of the two occurring forces on the examined object. Therefore, the third axiom ,precedes" the second axiom.

THE FOURTH NEWTONIAN AXIOM. In fact it defines not as an axiom but rather as Corollary 1. and 2 .

Corollary 1. "A body acted on by [two] forces acting jointly describes the diagonal of a parallelogram in the same time in which it would describe the sides if the forces were acting separately.", I. Newton [1], p. 417.

In order to prove Corollary 1. Newton does not apply the previous axioms, but a logical reasoning that the forces are independent from each other; therefore, it is irrelevant which on which side of the parallelogram the body moves along first and second towards the endpoint of the diagonal. This reasoning uses the independence of forces, the attributes of which were not individually postulated by Newton. We also refer to the fact that the parallelogram rule is regarded as an axiom in linear vector spaces (see e.g. P. R. Halmos [2].

So Corollary 1. which is generally regarded as THE FOURTh NewTONian AXIOM (see e.g. Á. Budó [3]), includes two independent statements. On the one hand, it states that the effect of forces acting upon a body are independent, on the other hand, they sum up as vectors. Since the Newtonian axioms apply to (point like) bodies, forces act upon them in one point; there are no conceptual obstacles for the vectors of forces to sum up.

Similarly to the summation of forces, Newton states in an individual Corollary 2. that a force can be separated to two oblique forces.

Corollary 2. "And hence the composition of a direct force $\mathrm{AD}$ out of any oblique forces $\mathrm{AB}$ and $\mathrm{BD}$ is evident, and conversely the resolution of any direct force $\mathrm{AD}$ into any oblique forces $\mathrm{AB}$ and $\mathrm{BD}$. And this kind of composition and resolution is indeed abundantly confirmed from mechanics.", I. Newton [1], p. 418.

In order to prove this, Newton used the condition torque of forces on one point and the balance of parallel forces placed on two opposite ends of the balance beam of the scale.

The following statements can be made in connection with Newtonian axioms:

The axioms do not include information regarding the occurrence of force, i.e. the axioms do not determine the generation of force.

The second axiom, more specifically its definition in the form of $\mathrm{F}=\mathrm{ma}$, enables the measurement of mass and weight force (see e.g. Á. Budó [3]).

The axioms apply to the motion of bodies that can be

*Corresponding author: glamer@eng.unideb.hu 
described with shifting. Instead of (point like) bodies, they can also be regarded as extensive objects (for instance rigid or deformable solid bodies), but occurring forces only act in the centre of gravity and the bodies cannot rotate around their centre of gravity.

The axioms do not deal with the movement of extensive, e.g. rigid or deformable solid, bodies. These have to be examined separately.

The axioms do not refer to equilibrium and the effect of the equilibrium force systems on motion.

The axioms do not apply to the rotation, deformation and fracture of bodies.

The axioms do not include the occurrence of constraint forces, and neither the fact that in case of a constraint created by a surface, the constraint force is orthogonal to the surface.

Newton's axioms primarily apply to the motion of bodies, i.e. the followings are excluded: deformation of bodies, fracture of bodies, equilibrium of bodies.

\section{Axioms of statics}

The axioms of statics apply to the equilibrium of rigid bodies (see e.g. K. Szily [4] and T. Cholnoky [5]).

Before presenting the axioms, force, body and the mechanical state to which they apply must be defined.

Force: effect occurs while two bodies act upon each other. Force is characterised by four data: point of application, line of action, magnitude and sign.

Characterisation of the body: A body is an identifiable collection of matter which moves together, the distance between any two points of the body are constant and the collection of particles remains. Generally, the rigid body is regarded as a three dimensional, finite volume region in the Euclidean space which does not change its size upon forces.

Characterisation of equilibrium: a body is motionless in equilibrium.

The axioms of statics are the following.

THE FIRST AXIOM OF STATICS: Two forces of identical magnitude and line of action, with opposite sign are in equilibrium.

This axiom captures, by providing two forces in equilibrium, that equilibrium force systems exist.

This axiom defines the equilibrium of two forces irrespective of point of application: the point of application of forces is simply not included among the conditions of equilibrium. Consequently, two forces do not have to act upon in the same point; the condition of equilibrium only depends on concordance of line of action (beyond the identical magnitude and opposite sign).

THE SECOND AXIOM OF STATICS: Three forces are only in equilibrium if the lines of action of three forces intersect in one point, and the vector of three forces constitute a closed, continuous vector polygon (triangle).

The axiom implicitly applies the fact that the force vectors are present as independent entities, as well as the fact that they can be summed as vectors. These are conditions for identifying the sum of three force vectors with the force equal that of the simultaneous effect of three forces.

The closed, continuous vector polygon indicates that the three forces must be in one plane.

THE THIRD AXIOM OF STATICS: If we connect an equilibrium force system to another equilibrium force system or an equilibrium subsystem is taken away from an equilibrium system, the obtained force system remains in equilibrium.

This axiom states how another equilibrium force system can be obtained from another equilibrium force system. Only a strictly equilibrium force system can be used to create an equilibrium force system: either two equilibrium force systems are ,added”, or a partial equilibrium force system constituting a part of the equilibrium force system is ,subtracted”. Equilibrium force system cannot be created from non-equilibrium force system. This is defined as equilibrium and nonequilibrium force systems are independent from each other.

THE FOURTH AXIOM OF STATICS: If forces arise in pairs when two rigid bodies act upon each other, the pair of forces have the same line of action, the same magnitude and opposite sign.

This axiom defines only that forces arise in pairs. It does not include information on either the number of force pairs or the point of application occurring with force pairs and neither does it refer to whether the two rigid bodies are motionless or are in motion in the moment of occurrence of force. It does not specify whether motionless bodies begin to move when force pairs arise or do the arising forces change motion in case of moving bodies or not.

The two forces, in THE FOURTH AXIOM OF STATICS, between two rigid bodies occurring as a consequence of an effect, are in equilibrium according to THE FIRST AXIOM OF STATICS.

The following statements can be made regarding the axioms of statics:

The axioms do not include information on the occurrence of forces, i.e. the axioms do not determine the occurrence of forces.

The axioms are formally applied to the equilibrium of rigid bodies. In fact, the first three axioms of statics include conditions suitable for determining equilibrium irrespective of the fact whether forces act upon rigid or deformable bodies or the forces are simply given in space. The fourth axiom of statics includes reference to rigid bodies, as long as the aforementioned axiom makes constraints regarding the force pairs occurring during the interaction of two rigid bodies.

The axioms do not refer to motion and the effect of motion on the equilibrium force system.

The axioms do not deal with the effect of forces occurring between bodies on the state of equilibrium and motion.

The axioms do not apply to the motion (shifting), rotation, deformation and fracture of bodies.

* Corresponding author: glamer@eng.unideb.hu 
The axioms do not include the development of constraint forces or the fact that the constraint force is orthogonal to the surface in case of constraint created by a surface.

The axioms of statics are primarily applied to rigid bodies at rest, i.e. exclude: deformation of bodies, fracture of bodies, the motion of bodies.

We examine such tasks within statics, where the system of motionless, rigid bodies in equilibrium rearrange due to the effect of a given force system and reach a motionless state of equilibrium, but the path to this is imagined as the rearrangement of rigid body systems occurs without motion until equilibrium is reached. Bodies do not gain either velocity or acceleration during the rearrangement. Equilibrium is reached infinitely slowly.

\section{Criteria of establishing the axiom system}

The behaviour of bodies under forces is determined based on knowledge through experience, and from them abstracted fundamental theorems that is axioms.

We follow the following principles when setting up axiom system:

The axioms must record

1) how the force occurs,

2) what data describe (characterise) the force,

3) how the occurring force(s) can be described,

4) what the effect of two forces on each other like,

5) what mathematical object force is,

6) what state exists if there is force and if there is no force,

7) how the forces characterising a mechanical system (equilibrium force system) can be determined in case of an ,elementary”, or a ,typical" system,

8) how can be created or derived from one specific (let understand: equilibrium) force system an another specific (that is equilibrium) system, different from beginning one.

The axioms are classified into four large groups.

The first group summarises the knowledge regarding the occurrence of forces. The second group includes interpretations of forces as mathematical objects. The third group records the effect of forces on bodies. The fourth group describes the relationship between forces and their effects.

\section{A complete axiom system related to forces}

The axioms are classified into four main groups: occurrence of force, force as mathematical object, effect of force on bodies, relationship between force and its effects.

\section{B. Axioms related to the occurrence of force}

The first axiom records how force occurs.

THE FIRST AXIOM (The axiom of interpretation of force): Force is the mechanical model of two interacting bodies. An effect occurring between two bodies is modelled as two forces: one body acts upon another body and vice versa, the other body acts upon one body. So when two bodies act upon each other, in case of one kind of effect, a pair of forces occur. In case of various effects, forces occur in pairs when two bodies act upon each other; as many pairs as many effects occur between the two bodies.

With two given bodies, $A$ and $B$, and an existing effect between them (e.g. they are in contact in a point, or gravitational pull exists between them). The effect of body $B$ on body $A$ is denoted by $F_{A B}$, the effect of body $A$ on body $B$ is denoted as $F_{B A}$. Since THE FIRST AXIOM only records that there is an effect, so it is denoted by a „neutral" notation, by letter $F$. If there are more effects between body $A$ and $B$ (e.g. there are contact in several points, or gravitational and electrical attraction, and repulsion also exists between them), then the forces occurring in pair differ from each other in accordance with more effects; $F_{A B}^{1}$ and $F_{B A}^{1}$, as well as $F_{A B}^{2}$ and $F_{B A}^{2}$ the forces occurring in contact point 1 and 2 , and $F_{A B}^{G}$ and $F_{B A}^{G}$, as well as $F_{A B}^{E}$ and $F_{B A}^{E}$ the forces occurring as an effect of gravitational or electrical attraction (repulsion).

The second axiom records the data characterising the force:

THE SECOND AXIOM (The axiom of data of force): Force is characterised by the point of application, line of action, scale and direction.

We characterise the occurring forces in the third axiom.

THE THIRD AXIOM (The axiom of action and reaction): The two forces occurring between two bodies as a result of effects between two bodies are characterised as follows: the line of action of two forces is in coincidence, the magnitude of two forces is the same, and their singe is opposite. We say that there is an ,action" and a ,reaction” occurring for each effect between two bodies.

THE THIRD AXIOM does not have ,provision” on the point of application of forces. The point of application is interpreted at different points in various modelling. For example in the case of gravitational bodies, gravitating force is connected to the centre of gravity of the gravitating body. Forces arising during the gravitational effect are far from each other in celestial body revolving around a central celestial body. Contact forces are in the same contact points in the case of contacting bodies.

It must be recorded how a force arising in the absence and presence of another force or another forces.

The fourth axiom records the method of effects of two or more forces, more specifically the independence of two or more forces from each other.

THE FOURTH AXIOM (The axiom of independence of forces): The individual forces act upon independently.

Let given three bodies, $A, B$ and $C$. The effect of body $B$ on body $A$ when there is no body $C$, denoting by

Corresponding author: glamer@eng.unideb.hu 
$F_{A B}(\varnothing)$, and $F_{A B}(C)$, if there is the body $C$ present, then, when body $A$ and $B$ act upon each other. The independence of forces means that $F_{A B}(\varnothing)$ is identical with $F_{A B}(C)$, i.e. the effect of body $A$ and $B$ on each other is not influenced by body $C$. Naturally, the axiom also applies to cases when more $C_{i}(i=1,2, \ldots, n)$ bodies are also present beyond body $A$ and $B$. On this case, the $F_{A B}(\varnothing)$ force arising without the $C_{i}(i=1,2, \ldots, n)$ bodies is identical with the $F_{A B}\left(C_{i}\right)(i=1,2, \ldots, n)$ force arising in the presence of any $C_{i}(i=1,2, \ldots, n)$ body, with the $F_{A B}\left(C_{i}, C_{j}\right)(i, j=1,2, \ldots, n, i \neq j)$ force arising along with any two, $C_{i}$ and $C_{j}(i, j=1,2, \ldots, n, i \neq j)$ bodies and with the $F_{A B}\left(C_{i}, C_{j}, \ldots, C_{l}\right)(i \neq j, i \neq l, \ldots, j \neq l, i, j, \ldots, l=$ $1,2, \ldots, n)$ forces arising along with any combination of bodies $\left.C_{i}, C_{j}, \ldots, C_{l}\right)(i \neq j, i \neq l, \ldots, j \neq l, i, j, \ldots, l=$ $1,2, \ldots, n)$.

\section{Force as a mathematical object.}

The fifth axiom records what mathematical object is force.

THE FIFTH AXIOM (The axiom of choosing the mathematical object): Force is a vector quantity.

As this axiom records that effect is modelled as a vector, effect - the force - is hereinafter denoted by a bold letter.

With the occurrence of two or more effects, i.e. two or force occurrence the fact must be recorded that if two or more forces are simultaneously interpreted, how those jointly act upon the body. This is recorded in the sixth axiom.

THE SIXTH AXIOM (The axiom of joint effect of forces): Forces with identical point of application acting upon independently sum up as vectors.

THE SIXTH AXIOM states that two forces with identical point of application sum up as vectors: their joint effects - their geometrical attributes - can be determined by vector addition. The point of application of the „summed" force is the common point of application of forces, line of action and magnitude is determined based on the rules of vector addition.

THE SIXTH AXIOM does not apply to the determination of joint effects by forces with different point of application. This requires individual examination.

\section{The effect of force on bodies: motion and} immobility

The following possibilities have to be separated in this section

1) No force acts upon the body

2) One force acts upon the body and no constraint exists

3) More forces act upon the body, no constraint exists and the body is in a state of inertia: the force system is in equilibrium (definition)

4) The existence of equilibrium force system

5) If there is a given equilibrium force system, how another equilibrium force system can be created from it 6) A force acts upon the body and one constraint exists

The seventh axiom records the fact that a body is in a motionless state without force.
Immobility or motion can only be interpreted compared to one or more precisely to three other bodies. Bodies providing the point of comparison can be motionless or can be in motion. Therefore new providing bodies of comparison have to be chosen. Then it must be decided whether the new (secondly chosen) providing bodies of comparison to determine the immobility of (firstly chosen) bodies are motionless or are in motion (compared to the older group of bodies). The selection of bodies for comparison to determine immobility or motion can be continued infinitely. This can only be eliminated if absolutely motionless bodies are chosen. This latter does not exist, as everything in the Universe is in motion. What remains is the system of bodies that can be regarded as practically motionless. This will simply be referred to as a motionless environment. This is what Newton refers to as system of inertia, as a system of absolute rest. As there is no information on motion (yet), immobility is regarded as a priority status. The equivalence of immobility and motion with uniform velocity can be interpreted later.

THE SEVENTH AXIOM (The axiom of immobility): A body without any forces acting upon it are at rest (compared to the environment being at rest).

This axiom refers to the state when no forces are acting upon the body; in other words no other bodies are acting upon it. A separate axiom states if a force acts upon the body. At the same time, not only one force can act upon the body, but also a support opposing the motion of its points.

DEFINITION: A body opposing the motion of some points of another body is called a constraint (support).

The effect of forces acting upon a body has to be examined separately for instances of lack of constraint and presence of constraint.

THE EIGHTH AXIOM (The axiom of motion): A body, upon which force is acted, and is not under the effect of constraint (compared to the motionless environment) does not remain motionless, it gains motion.

This axiom only records the fact that bodies without constraint start to move. The "how" of motion - depending on the type of body - can be recorded by some individual axioms.

In the presence of constraint(s), forces arising from the constraint(s) also act upon the body. This fact is recorded by the eighth axiom.

THE NINTH AXIOM (The axiom of arising support force). In body which is acted upon by a force and is under the effect of constraint, a force arises, which is opposite the direction of the constrained motion.

DEFINITION: If a motionless body is not under the effect of a constraint and is under more forces act upon it, remains motionless then the force system acting upon it are referred to as equilibrium force system.

Using the term of equilibrium force system the mechanical state of body under forces and constraints can be defined.

CONSEQUENCE 1: A body, which is under the effect of more forces and constraint(s) and the forces as well the

Corresponding author: glamer@eng.unideb.hu 
constraint(s) jointly

1) form an equilibrium force system, then the body is motionless,

2) do not form an equilibrium force system, then the body begins to move.

We provide the required and sufficient condition of equilibrium for two forces in the ninth axiom.

THE TENTH AXIOM (The axiom of equilibrium for two forces; the axiom of existence of equilibrium force systems): Two forces with identical line of action, identical magnitude and opposite sign are in equilibrium.

THE TENTH AXIOM records that an equilibrium force system exists by providing two forces in equilibrium.

THE TENTH AXIOM determines the equilibrium of two forces irrespective of point of application: the points of application simply are not present among the conditions of equilibrium. Consequently, two forces do not have to act upon in the same point, the condition of equilibrium only depends on the concordance of the lines action (beyond the identical magnitude and opposite sing).

A consequence of THE TENTH AXIOM, is that the two forces arising during action-reaction between two bodies are in equilibrium. The two bodies however, do not have to be in equilibrium; see e.g. the gravitational force among moving planets and the Sun. The fact that the two forces arising during action-reaction are in equilibrium can also be defined as the $\mathbf{F}_{A B}$ and $\mathbf{F}_{B A}$ forces arising during the interaction of $A$ and $B$ bodies are opposite each of other.

The eleventh axiom records the method creating an equilibrium force system from another equilibrium force system.

THE ELEVENTH AXIOM (The principle (axiom) of superposition of equilibrium force systems). If an equilibrium force system is connected to an equilibrium force system or an equilibrium subsystem is taken away from an equilibrium force system, then the obtained force system is still equilibrium.

THE ELEVENTH AXIOM states, how two equilibrium force systems, $\left\{\mathbf{A}_{i}\right\} \doteq\{\mathbf{0}\}$ and $\left\{\mathbf{B}_{j}\right\} \doteq\{\mathbf{0}\}$, can be used to create an equilibrium force system: the two independent equilibrium force systems are joined, i.e. the two joined force systems are regarded as one force system. The obtained force system is also in equilibrium: $\left\{\left\{\mathbf{A}_{i}\right\},\left\{\mathbf{B}_{j}\right\}\right\} \doteq\{\mathbf{0}\}$. Furthermore, THE ELEVENTH AXIOM states that if there is an equilibrium force system, $\left\{\mathbf{C}_{i}\right\}$ $\doteq\{\mathbf{0}\}$, which can be separate into two subsystems in a way that one is in equilibrium, $\left\{\mathbf{C}_{i}\right\} \doteq\left\{\left\{\mathbf{A}_{i}\right\},\left\{\mathbf{B}_{j}\right\}\right\}$ and $\left\{\mathbf{B}_{j}\right\} \doteq\{\mathbf{0}\}$, then leaving the equilibrium subsystem out of the original equilibrium force system, the remaining subsystem is still in equilibrium: $\left\{\mathbf{A}_{i}\right\} \doteq\{\mathbf{0}\}$.

The eleventh axiom records that the state of equilibrium and motion are independent mechanical states.

THE TWELFTH AXIOM (The axiom of independence of equilibrium and motion): If an equilibrium force system acts upon a body in motion, then the motion of the body is not influenced by an equilibrium force system and vice versa.

Note. THE TWELFTH AXIOM records the independence of motion and equilibrium. At the same time, it does not separate what mechanical phenomenon occurs in equilibrium: contact, deformation, rearrangement, friction etc. This will be defined by the laws related to determining force. At least must be recorded that force arising in equilibrium must be arisen infinitely slowly and dos not acts velocity and acceleration. This is formulated in the twelfth axiom.

THE THIRTEENTH AXIOM (The axiom of effect of equilibrium force): The forces arising in equilibrium must be arisen infinitely slowly, and dos not act velocity and acceleration.

\section{E. The relationship existing between force and its ef-} fect

The following axioms describe the relationship among various forces and their effects:

THE FOURTEENTH AXIOM (The axiom of gravitational forces). Force arising between two gravitating bodies: $\mathbf{F}=-\gamma \frac{m_{1} m_{2}}{r^{2}} \frac{\mathbf{r}}{r}$, where $m_{1}$ and $m_{2}$ are the mass of the two bodies, $\mathbf{r}$ is the position vector connecting two points of the centre of gravity, $\gamma$ is the universal gravitational constant.

THE FIFTEENTH AXIOM (The axiom of weight force): The weight force acting upon bodies with weight is: $\mathbf{G}=m \mathbf{g}$, where $m$ is the mass of the body, and $\mathbf{g}$ is the gravitational acceleration on the surface of the Earth.

THE SIXTEENTH AXIOM (The axiom of constraint force): A force arises at a contact point in a body under constraint is orthogonal to the surface of constraint. Its magnitude can be determined from the conditions of equilibrium.

THE SEVENTEENTH AXIOM (The axiom of friction force): A force arises at a connection point in a body under constraint with friction is tangent to the surface of constraint. Its magnitude depends on the normal force arising between body and surface, as well as the friction constant characterising friction surfaces.

\section{References}

[1] I. Newton: The Principia. Mathematical Principles of Natural Philosophy, University of California Press, Berkeley - Los Angeles - London, 1999. A new translation by B. Cohen and A. Whitman assisted by J. Budenz

[2] P. R. Halmos: Finite-dimensional vector spaces, Springer-Verlag, 1974.

[3] Á. Budó: Mechanika. (Mechanics in Hungarian) 5. kiadás. Tankönyvkiadó, Budapest, 1972.

[4] K. Szily: Mechanika (Mechanics in Hungarian). Első kötet. Statika. Németh József technikai Könyvkiadó Vállalata, Budapest, 1921.

[5] T. Cholnoky: Mechanika. (Mechanics in Hungarian) I. kötet. Statika. Harmadik, átdolgozott kiadás. Tankönyvkiadó, Budapest, 1969.

Corresponding author: glamer@eng.unideb.hu 